\title{
Les simplifications instrumentales et processuelles : un dispositif de gestion des tensions de gouvernance publique à l'Université
}

\author{
Laurent Mériade \\ Maître de conférences en Sciences de Gestion - CRCGM - Université d'Auvergne - IUT GEA \\ (meriadelaurent@gmail.com, tél : 0033675819028)
}

\section{Résumé}

Depuis la LOLF (2001), la LRU (2007) puis la RGPP (2008), les universités françaises se sont retrouvées au centre d'injonctions paradoxales imposant une gestion spécifique de tensions de gouvernance de publique (Bartoli et al., 2012).

Alors que la littérature décrit la gestion de ces tensions plutôt par des instruments discursifs ou comportementaux (Mazouz et al., 2012, Huddon et Mazouz, 2015), notre objectif de recherche vise à révéler, à travers l'exemple des universités françaises, le rôle des indicateurs de performance dans la gestion de ces tensions.

Une étude comparative de données issues de trois niveaux de production d'indicateurs de performance pour les universités françaises nous permet d'illustrer cette analyse en identifiant et caractérisant des simplifications instrumentales et processuelles destinées à gérer ces tensions.

Mots clés. Simplifications, tensions, université, injonctions paradoxales.

\begin{abstract}
Since the LOLF (2001), the LRU (2007) and the RGPP (2008), French universities have found themselves at the center of paradoxical injunctions requiring a specific management of public governance tensions (Bartoli et al., 2012).

While the literature describes the management of these tensions rather by discursive or behavioral instruments (Mazouz et al., 2012 Huddon and Mazouz, 2015), our research objective is to reveal and to characterize the management tools of these tensions in French universities.

A comparative study of data from three levels of production performance indicators for French universities allows us to illustrate this analysis by identifying and characterizing instrumental and procedural simplifications for managing these tensions.
\end{abstract}

Keywords. Simplifications, tensions, university, paradoxical injunctions. 


\section{Introduction}

Dans les organisations publiques, les nouveaux objectifs inspirés par le New Public Management (NPM) visent l'amélioration de la rentabilité et de la satisfaction des citoyens associée à une diminution des règles et procédures (Bartoli, 2008). L'application de ces préceptes s'est accompagnée, en France notamment, d'un important développement d'outils de gestion centrés sur les résultats (Mazouz et Tremblay, 2006). Ceci a fait parfois naître, au sein de nombreuses organisations publiques, des réflexes de simplification de la complexité de ces outils.

De même, la situation d'apparente liberté pour organiser les processus publics provoque un certain nombre de tensions de gouvernance publique (Bartoli et al, 2012 ; Facal et Mazouz, 2014) autour de l'injonction paradoxale «moins de procédures pour plus de résultats ».

Comment les managers publics gèrent-ils ces tensions de gouvernance à travers les instruments de gestion qu'ils mobilisent et qu'ils s'approprient?

Pour se simplifier administrativement, les administrations publiques sont souvent tenues de se complexifier sur le plan managérial. Ainsi, la simplification administrative va s'accompagner souvent d'une complexification des processus, au niveau du «back-office » (Fixari et Pallez, 2007). Ceci nécessitera en retour des simplifications processuelles afin de gérer les tensions de gouvernance créées par la recherche d'objectifs parfois asymétriques (performance financière et amélioration du service à l'usager, performance publique et diminution du front office, performance sociale et augmentation des interactions usagers/agents publics) (Poel et al. 2014).

Depuis la $\operatorname{LOLF}^{1}$ (2001), la LRU² (2007) puis la RGPP ${ }^{3}$ (2008), les universités françaises se sont retrouvées au centre de réformes porteuses d'objectifs contradictoires (par exemple, rationaliser les dépenses et améliorer les services). La multiplication des injonctions paradoxales de ce genre impose de la part des acteurs publics une gestion spécifique des tensions de gouvernance que font notamment naître les mesures de simplification administrative et les instruments de gestion qui s'y rapportent.

Brunsson (2002) qualifie d'hypocrisie organisationnelle l'ensemble des tensions et contradictions entre discours, décisions et actions que doit gérer l'organisation en manipulant plusieurs valeurs contradictoires simultanément. La littérature en management public décrit la gestion des tensions de gouvernance publique plutôt par des instruments discursifs ou comportementaux (Bartoli et al., op.cit., Mazouz et al., 2012, Huddon et Mazouz, 2015). Notre objectif de recherche, à travers l'exemple des universités françaises, vise à révéler et à caractériser le rôle des indicateurs de performance dans la gestion de ces tensions.

Afin de soutenir cette proposition, notre travail s'organise en trois temps. Dans un premier temps, nous revenons sur les avancées de la littérature en matière de pilotage de la performance des universités afin de justifier l'intérêt de la mobilisation du cadre conceptuel de l'hypocrisie organisationnelle (Brunsson, 1993, 2002) pour compléter ces approches. Par la suite, après avoir précisé la méthodologie utilisée, nous analysons les principaux indicateurs de performance qui environnement les universités françaises, puis nous mettons en évidence les tensions de gouvernance qu'ils provoquent et les simplifications qui sont opérées pour les gérer.

\section{Revue de littérature et approche conceptuelle}

En France, la LOLF a notamment introduit une obligation de performance dans la gestion financière des universités (Mignot-Girard, 2008) selon un mode d'évaluation intégrant des indicateurs de performance d'abord définis par des programmes ministériels et des P.A.P. ${ }^{4}$ spécifiques. Elle a contribué, plus spécifiquement dans le domaine universitaire, à la construction de budgets orientés vers des cibles de performance définies notamment à partir des programmes 150 (Formation et

\footnotetext{
${ }^{1}$ Loi Organique sur les Lois de Finances

${ }^{2}$ Loi Libertés et Responsabilités des Universités

${ }^{3}$ Révision Générale des Politiques Publiques

${ }^{4}$ Projet Annuel de Performance
} 
recherche universitaire) et 231 (Vie étudiante) de la MIRES 5 .

$\mathrm{La}$ LRU et le passage aux $\mathrm{RCE}^{6}$ ont accordé de nouvelles responsabilités aux directions universitaires, par contre, ces deux dispositifs ont rarement précisé comment ces nouvelles responsabilités budgétaires devaient s'exécuter. Dès lors, ces dernières s'opèrent à partir de mesures de gestion dont l'initiative est laissée aux organes de direction universitaire.

La LRU, venant à la suite de la mise en œuvre de la LOLF, a permis de porter la logique managériale du NMP en modifiant significativement la réflexion stratégique et le pilotage budgétaire (Boitier et Rivière, 2013). Le passage aux RCE (à partir de 2009) a élargi le rôle des instances de direction universitaire dans le pilotage budgétaire en leur attribuant la mission de développer des procédures de dialogue budgétaires dans la gestion des relations entre l'Université et ses composantes (ibid.)

Ezzamel et al. (2012), à la suite de Reay et Hinings (2009), notent que ces mesures constituent des instruments permettant de gérer la concurrence entre les principales logiques qui traversent les organisations complexes comme l'Université (logiques professionnelle, managériale et politique). Moll et Hoque (2011) démontrent que les logiques politique et professionnelle restent très influentes et attribuent aux indicateurs un rôle prééminent de gestion des conflits de rationalité et de légitimation des décisions de la direction (Mériade, 2013). Cette gouvernance à la fois professionnelle et politique des universités (Fabre, 2013) provoque une sélection d'indicateurs de gestion en mesure de prendre en compte des éléments de négociation et de discussion à deux niveaux (niveau local avec les composantes et niveau central avec le Ministère). Cette sélection se réalise à des niveaux distincts d'instrumentation qui affinent et simplifient le choix des instruments de gestion quotidienne des universités (Mériade et Mainetti, 2013).

Carassus et al (2012) identifient trois explications essentielles à l'apparition de ces simplifications qu'ils qualifient de déviances organisationnelles: des conflits de contexte, de spécificités des organisations et un manque d'information des acteurs. Il nous semble important d'identifier le plus justement possible l'origine de ces déviances qui naissent souvent de tensions de gouvernance publique repérables dans les usages ou les pratiques de gestion.

Dans cette perspective, Mazouz et Tardif (2009) recensent quatre niveaux d'étude des tensions de gouvernance publique qui portent sur les institutions, l'organisation, le cadre de gestion, et les outils de gestion à proprement parler. L'étude de ces tensions de gouvernance s'est jusqu'alors particulièrement concentrée sur celles relatives à l'éthique dans l'accomplissement des services publics (Bartoli et al, op.cit.; Chomienne et al., 2012). A l'Université, comme dans probablement d'autres institutions publiques, ces tensions se formalisent aussi à travers des simplifications des outils de gestion ou de pilotage dont il est difficile d'évaluer le caractère volontariste. L'outil de gestion étant intrinsèquement déviant (Lorino, 2007), nous préférons insister sur les raisons de l'apparition de ces simplifications qui tiennent souvent à leurs capacités à faire disparaître ou à gérer des tensions de gouvernance.

Ces simplifications sont l'expression des paradoxes de l'instrumentation de gestion dans les universités (Dreveton et al., 2012) qui se traduisent, par exemple, par la sélection d'indicateurs plutôt financiers alors même que les objectifs de performance sont explicitement définis par des indicateurs non financiers (nombre d'étudiants présents aux examens, nombre d'étudiants boursiers, nombre d'enseignants-chercheurs publiants, taux d'occupation des locaux, etc.).

L'approche de l'hypocrisie organisationnelle (Brunsson, 1992 ; 2002) participe à la compréhension des organisations et des paradoxes qui la traversent et peut nous aider à appréhender des tensions de gouvernance universitaire.

Pour Brunsson et Geoffroy (2012), l'hypocrisie organisationnelle permet de gérer les valeurs contradictoires qui naissent de cette coexistence. En présence de fortes incertitudes, les règles formelles ne peuvent pas suffire. Aussi, l'hypocrisie organisationnelle va apparaître à deux niveaux.

\footnotetext{
${ }^{5}$ Mission Interministérielle pour la Recherche et l'Enseignement Supérieur

${ }^{6}$ Responsabilités et Compétences Elargies
} 
Au niveau de l'organisation d'action qui va produire une idéologie organisationnelle informelle qui permet de rapprocher et uniformiser les valeurs et opinions des membres de l'organisation (Brunsson, 2002).

$\mathrm{Au}$ niveau de l'organisation politique qui cherche à créer de la légitimité vis-à-vis de l'environnement extérieur à travers les discours plus que les décisions. La construction de ces discours va se développer à partir des valeurs contradictoires et des conflits d'intérêts. L'existence de ces tensions contradictoires plutôt que de créer du désordre va favoriser la discussion et l'échange (Brunsson et Geoffroy, op.cit.).

Brunsson (2002) qualifie d'hypocrisie organisationnelle l'ensemble des tensions de gouvernance que manipule l'organisation et qui naît de trois types de contradictions :

(1) Les contradictions interindividuelles qui proviennent des conflits entre les individus qui imposent des négociations permanentes.

(2) Les contradictions entre les idées et les actions. Les idées et les discours étant moins coûteux en argent et en temps que l'action.

(3) Les contradictions entre les décisions et les actions dans la mesure où la rationalité de la décision pourra rentrer en conflit avec l'irrationalité de l'action (Brunsson, 1993 ; 2002).

L'hypocrisie organisationnelle plutôt que de résoudre ces tensions va les mobiliser pour permettre aux deux sphères d'action et politique de cohabiter. L'hypocrisie interne va générer de l'ambiguiité et ainsi permettra de contenter des intérêts divergents. L'hypocrisie externe va cultiver les contradictions entre les discours, les décisions et les actions afin de légitimer l'organisation tant sur le plan des valeurs (l'organisation politique) que de l'efficacité organisationnelle (l'organisation d'action).

Cependant, différentes formes de pilotage de l'hypocrisie organisationnelle peuvent apparaître compte tenu de la diversité et de la complexité des intérêts qui se superposent dans une organisation.

Une des faiblesses de l'approche de Brunsson provient de la grande diversité des formes d'apparition de l'hypocrisie organisationnelle. Elle peut provenir de la manipulation des contradictions et de leur exaltation, comme le soutient l'auteur, mais elle peut également apparaître sous d'autres formes en fonction notamment de la nature des discours, des actions et des décisions à l'origine des contradictions. Ceci est lié avec une autre faiblesse de l'approche de Brunsson qui provient de la difficulté à détecter dans les organisations l'hypocrisie organisationnelle.

Il nous semble que la mise en évidence des conséquences de l'hypocrisie organisationnelle peut s'opérer par repérage des tensions et des contradictions constitutives de cette hypocrisie. Nous postulons ici que les instruments de gestion dans les organisations publiques sont également des moyens de manager cette hypocrisie organisationnelle en gérant les tensions de gouvernance publique qui naissent des contradictions. Nous procédons pour cela à une analyse des zones et moments de contacts entre les indicateurs de performance et les organisations universitaires afin de mesurer leurs conséquences en termes de gestion des contradictions et des tensions de gouvernance.

\section{Méthodologie}

A partir du cadre conceptuel de l'hypocrisie organisationnelle, le repérage des indicateurs de performance universitaire en usage doit nous permettre de situer les tensions de gouvernance publique qui peuvent les générer. Une étude comparative des indicateurs de performance prescrits dans les P.A.P. (repris par 1'H.C.E.R.E.S. ${ }^{7}$ ) et par la D.G.E.S.I.P. ${ }^{8}$ avec les résultats d'une enquête sur les usages de ces indicateurs menée auprès des présidents de quarante-deux E.P.S.C.P. ${ }^{9}$ en France structure notre approche méthodologique. Pour cette dernière enquête, cinq questions fermées portaient sur les niveaux de performance perçus par les répondants et les indicateurs en mesure de les évaluer. Les réponses ont été analysées par un logiciel d'analyse statistique (SPSS Version 20.0) afin de réaliser une analyse en composante principale et de décrire les principaux

Haut Conseil à l'Evaluation de la Recherche et de l'Enseignement Supérieur

Direction Générale de l'Enseignement Supérieur et de l'Insertion Professionnelle

Établissement public à caractère scientifique, culturel et professionnel 
indicateurs de la performance identifiés par les présidents. Trois questions ouvertes portées sur les usages de ces indicateurs et ont été analysées sur SPSS à partir d'une catégorisation a posteriori des données afin de procéder à une caractérisation des indicateurs en usage.

L'ambition de cette première phase d'analyse était de mettre en lumière les différentes catégories d'indicateurs intervenant dans l'évaluation de la performance universitaire en France.

Dans un deuxième temps, nous confrontons ces catégories d'indicateurs de performance avec les formes de contradictions décrites par Brunsson $(1993,2002)$ et observables dans les universités. Pour cela, la mobilisation de notre cadre conceptuel nous permet de mettre en évidence les simplifications instrumentales réalisées au niveau des universités et de les justifier par des tensions de gouvernance publique (TGP, Bartoli et al. op.cit.) provenant des contradictions observables.

\section{Résultats}

L'instauration de nouveaux indicateurs de performance à l'Université est assez représentative d'une perturbation des représentations et des situations professionnelles des personnels (Berry, 1983). En redéfinissant la notion de performance notamment autour d'une simplification des relations usager/service public (Bartoli et Hermel, 2006), les nouveaux indicateurs perturbent les habitudes et processus traditionnels de travail (Boussard et Loriol, 2009). Ceci impose de la part des managers des adaptations qui prennent la forme de simplifications de l'outillage d'évaluation de la performance afin de gérer la « surcharge » et les « cercles vicieux bureaucratiques » (Crozier, 1964) facilement induits par un trop grand nombre d'indicateurs d'évaluation.

Dans un premier temps, nos premiers résultats présentent les trois niveaux de construction des indicateurs de performance (niveaux législatif, exécutif et organisationnel). Dans un deuxième temps, nous suggérons l'existence de deux niveaux de simplification des indicateurs de performance universitaire (simplification quantitative et simplification de sens) opérés notamment au niveau des établissements universitaires. Enfin, dans un troisième temps, nous décrivons le pouvoir justificatif de ces simplifications par les tensions de gouvernance publique qu'elles gèrent.

Brunsson (2002) ayant essentiellement observé des formes discursives d'hypocrisie organisationnelle, notre approche vise à démontrer qu'au-delà des discours ou des comportements, des simplifications instrumentales souvent peu visibles peuvent aussi constituer des moyens de gérer les tensions de gouvernance publique.

Dans le cadre de notre étude, ces simplifications peuvent être associées à un recentrage progressif de l'attention accordée à la performance universitaire autour d'objectifs ou d'indicateurs soit prescrit au niveau central, soit construit au niveau local. Il est possible de distinguer en effet au moins trois niveaux de production d'objectifs et indicateurs de performance universitaire.

\section{Le niveau législatif}

Un premier niveau qui se structure autour des indicateurs issus des objectifs et indicateurs votés annuellement par l'Assemblée Nationale dans le PLF (Projet de Loi de Finances) et se rapportant au programme 150 «Formations supérieures et recherche universitaire », principal programme dédié au Ministère de l'Enseignement Supérieur.

Ce niveau législatif de prescription a des effets sur les choix budgétaires des universités et des indicateurs de performance apparaissant au niveau des unités budgétaires (UB). Par exemple, en matière de recherche, l'apparition de l'objectif «Montant des ressources sur titres de propriété intellectuelle » dans le PAP 150 de 2015 impose de la part des universités de privilégier des indicateurs et des activités permettant de construire des ressources propres provenant des activités de recherche. De même, toujours en 2015, l'apparition de l'objectif de performance «Renforcer l'ouverture européenne et internationale des établissements » incite les universités à développer des partenariats internationaux tant sur le plan scientifique que pédagogique. De même, en matière de formation, l'inscription de l'objectif «Améliorer la réussite des étudiants » dans ce même PAP peut influencer, de manière significative, le choix des formations proposées et les modalités de contrôle des connaissances.

La prescription de ces indicateurs à ce niveau influence significativement les choix des indicateurs 
qui sont opérés, par la suite, aux niveaux du Ministère puis des universités.

\section{Le niveau exécutif}

Un deuxième niveau d'objectifs et indicateurs de performance est prescrit par le modèle d'attribution des moyens («modèle Sympa ») défini par la DGESIP du Ministère de l'Enseignement Supérieur. Ce modèle est fondé sur deux types d'indicateur (indicateur d'activité et indicateur de performance) afin de calculer la dotation annuelle de moyens attribuée à chacune des universités françaises. Ces indicateurs portent, d'une part, sur la formation et, d'autre part, sur la recherche et déterminent, de manière significative, les politiques internes des universités notamment au niveau de la gestion des moyens. Les indicateurs développés par ce modèle depuis 2009 et les changements d'indicateurs intervenus pour la construction des budgets des universités pour 2015 (tableau $n^{\circ} 1$ et tableau ${ }^{\circ} 2$ ) modifient le pilotage de la performance des universités dans la mesure où les nouveaux indicateurs ou leurs nouveaux modes de calcul impactent les choix des indicateurs locaux.

Par exemple, en 2015, l'apparition dans le «modèle Sympa » des indicateurs « taux d'étudiants en apprentissage » ou « nombre d'heures stagiaires » incitent à privilégier des formations en mesure de renforcer l'autonomie financière des universités.

De même, en matière de recherche, l'apparition des indicateurs financiers relatifs au "montant des contributions des projets des enseignants chercheurs » tend à privilégier la contribution financière des enseignants-chercheurs plutôt que leurs apports scientifiques (disparition de la notion de « chercheurs produisants »).

Par la suite, le choix de ces indicateurs financiers et quantitatifs se répercute directement au niveau de l'organisation des universités et des catégories d'indicateurs de performance privilégiées.

Tableau $n^{\circ} 1$ : Evolution indicateurs de formation Sympa (Source : DGESIP)

\begin{tabular}{|c|c|c|}
\hline FORMLATION & Indicateurs Sympa 2009 & \begin{tabular}{|l} 
Indicateurs Sympa 2015 \\
\end{tabular} \\
\hline \multirow{3}{*}{$\begin{array}{l}\text { Indicateurs } \\
\text { d'activité }\end{array}$} & $\begin{array}{l}\text { nombre d'étudiants inscrits présents } \\
\text { aux examens pondérés par classe de } \\
\text { formation, pour les niveaux L et M }\end{array}$ & $\begin{array}{l}\text { Nombre d'étudiants présents aux } \\
\text { examens pondérés par classe de } \\
\text { formation }\end{array}$ \\
\hline & $\begin{array}{l}\text { enveloppe spécifique « plan } \\
\text { licence », nombre d'étudiants en L }\end{array}$ & ( \\
\hline & $\begin{array}{l}\text { enveloppe spécifique } \\
\text { « compensation TD }=\mathrm{TP}\end{array}$ & - \\
\hline \multirow{5}{*}{$\begin{array}{l}\text { Indicateurs } \\
\text { d'incitation } \\
\text { (ou de } \\
\text { performance) }\end{array}$} & nombre de diplômés de $\mathrm{M}$ & nombre de diplômés de $\mathrm{L}$ et $\mathrm{M}$ \\
\hline & $\begin{array}{l}\text { valeur ajoutée de la réussite en L et } \\
\text { DUT }\end{array}$ & $\begin{array}{l}\text { valeur ajoutée de la réussite en } \mathrm{L}, \mathrm{LP} \\
\text { DUT et } \mathrm{M}\end{array}$ \\
\hline & \begin{tabular}{|c|}
- \\
\end{tabular} & taux d'étudiants en apprentissage \\
\hline & - & $\begin{array}{l}\text { nombre d'heures stagiaires } \\
\text { enseignants }\end{array}$ \\
\hline & - & $\begin{array}{l}\text { taux d'inscrits titulaires d'un bac techno } \\
\text { ou pro en lre année de DUT }\end{array}$ \\
\hline
\end{tabular}


Tableau $n^{\circ} 2$ : Evolution indicateurs de recherche Sympa (Source DGESIP)

\begin{tabular}{|c|c|c|}
\hline RECHERCHE & Indicateurs Sympa 2009 & Indicateurs modèle 2015 \\
\hline $\begin{array}{c}\text { Indicateur } \\
\text { d'activité }\end{array}$ & $\begin{array}{l}\text { nombre d'enseignants- } \\
\text { chercheurs « produisants » }\end{array}$ & $\begin{array}{l}\text { le nombre d'enseignants-chercheurs } \\
\text { environnés }\end{array}$ \\
\hline \multirow{4}{*}{$\begin{array}{l}\text { Indicateurs } \\
\text { d'incitation (ou } \\
\text { de } \\
\text { performance) }\end{array}$} & cotation des laboratoires & - \\
\hline & nombre de thèses soutenues & $\begin{array}{l}\text { nombre de thèses de doctorat soutenues } \\
\text { (dont le nombre de thèses d'exercice en } \\
\text { santé pondéré à } 1 / 2 \text { ) }\end{array}$ \\
\hline & - & $\begin{array}{l}\text { pourcentage d'enseignants-chercheurs } \\
\text { membres actifs de l'IUF }\end{array}$ \\
\hline & - & $\begin{array}{l}\text { Montant des contributions part } \\
\text { enseignant-chercheur des projets déposés } \\
\text { et évalués du 7e PCRDT }\end{array}$ \\
\hline
\end{tabular}

\section{Le niveau organisationnel}

Un troisième niveau d'apparition d'objectifs et indicateurs de performance se situe au niveau des tableaux de bord et de pilotage développés dans les universités et inspirés par les contrats quinquennaux entre l'État et les universités. Ce niveau révèle les choix de variables à surveiller opérés par les directions universitaires.

Dans le cadre de notre enquête auprès des présidents d'université, nos analyses mettent en évidence deux catégories principales d'indicateur définies par les présidents comme essentielles au pilotage de leur université (indicateurs de pilotage des formations et des moyens). Ces deux catégories naissent de l'appropriation par les universités des indicateurs des niveaux législatif et exécutif. Cette appropriation se construit en réponse à des tensions de gestion et de gouvernance qui se développent au point de rencontre de ces indicateurs et des divers intérêts et rationalités existants dans les universités.

A ce niveau organisationnel, l'appropriation se concrétise par la simplification des indicateurs de performance autour de ces deux catégories (formation et moyens) en privilégiant des indicateurs financiers et quantitatifs (par exemple, maitrise de la masse salariale, taux de réussite des étudiants, taux d'insertion professionnelle) plus consensuels que des indicateurs qualitatifs.

A partir de ces trois niveaux ou lieux de production des indicateurs de performance universitaire, nous mobilisons notre cadre de conceptuel afin de réaliser une analyse des simplifications opérées autour de ces indicateurs et de leurs thématiques (formation, recherche ou moyens). L'ambition initiale est de proposer une heuristique permettant de comparer et de mesurer l'impact de plusieurs niveaux de production d'indicateurs de performance sur les tensions de gouvernance observables au sein des universités françaises.

\section{Des simplifications des indicateurs pour gérer les tensions et contradictions}

Le modèle de Brunsson propose une description de la gestion des contradictions à partir des discours, des décisions ou encore des actions qui permettent de maintenir l'équilibre entre organisation d'action et politique en satisfaisant les intérêts divergents. Nous proposons de décrire les tensions et contradictions universitaires à travers les indicateurs de performance permettant la gestion de celles-ci. Cette gestion se caractérisant par des simplifications peu illustrées jusqu'ici par la littérature.

Les contradictions mises en évidence par le modèle de l'hypocrisie organisationnelle (Brunsson, 2002) constituent un point d'entrée fécond dans l'analyse des indicateurs de la performance universitaire. A partir de la comparaison de trois lieux de production de ces indicateurs (le niveau législatif, le niveau exécutif et le niveau organisationnel), ce modèle permet de mieux comprendre comment peuvent être gérées les tensions de gouvernance publique. En effet, il apparaît que face à ces tensions, les acteurs publics mettent en œuvre un certain nombre de simplifications 
instrumentales afin de gérer les tensions de gouvernance publique issues des controverses.

Nous identifions deux types de simplifications instrumentales :

- des simplifications quantitatives correspondant à des suppressions d'indicateurs

- des simplifications de sens correspondant à la substitution ou à la conversion de certains indicateurs

Nous proposons, par la suite, de justifier l'apparition de ces deux types de simplifications à partir de l'identification des tensions de gouvernance publique représentatives des contradictions entre discours, actions et décisions (Brunsson, 1993, 2002).

\section{Des simplifications quantitatives des indicateurs}

Tout en matière scientifique, les indicateurs de performance prescrits se justifient de plus en plus par des opérations financières et monétaires (recettes notamment) qui font naître des tensions de gouvernance autour d'un certain nombre d'indicateurs. Ainsi, les indicateurs relatifs au montant des contrats de recherche ou de contribution des enseignants chercheurs créent des tensions autour de la place de la recherche et de son indépendance (tableau $n^{\circ} 3$ ). Face à un certain corporatisme et dans un esprit de respect de la collégialité, les présidences universitaires semblent avoir fait le choix de ne pas utiliser ces indicateurs dans leurs outils de pilotage de la performance. Il est également possible de considérer que cette tension entre rentabilité et indépendance de la recherche puisse justifier également la suppression, dans les indicateurs à l'usage dans les universités, des indicateurs « nombre d'EC environnés » et « nombre de thèses soutenues » provenant du modèle Sympa.

Toujours en matière de recherche, la suppression des indicateurs "coopération internationale » et « nombre d'EC membres de l'IUF » répond là aussi à une tension entre réputation internationale et ancrage national ou régional des universités que les présidents d'université semblent gérer en privilégiant, pour la réputation de leurs universités, des indicateurs de performance fondés sur la réussite et l'insertion professionnelle de leurs étudiants.

En matière de formation, les suppressions des indicateurs "Pourcentage d'une classe d'âge obtenant un diplôme de l'enseignement supérieur » et «valeur ajoutée de la réussite » peuvent se justifier par une tension entre objectifs et mesure concrète de la qualité des services universitaires (tableau $n^{\circ} 3$ ). En effet, les PAP comme le modèle Sympa produisent ces indicateurs à partir de mesures contestées par un certain nombre d'université car ne prenant pas en compte toutes leurs spécificités. Ainsi les calculs de la "Valeur ajoutée» ou du "Pourcentage d'une classe d'âge obtenant un diplôme de l'enseignement supérieur » sont remis en cause car ils créent une tension entre qualité et lieux d'exercice du service public. En effet, il apparaît difficile pour certaines universités (parisiennes par exemple) de créer de la valeur ajoutée tant leurs publics peuvent être favorisés sur le plan socio-économique par rapport à des étudiants de province. De même, cette tension entre qualité et lieux d'exercice du service public provoque une contestation du calcul de la « qualité de la gestion immobilière » qui elle aussi est dépendante du lieu et des infrastructures existantes.

\section{Des simplifications de sens des indicateurs}

D'un autre côté, certains indicateurs non prescrits par les instances de tutelle apparaissent parmi les indicateurs mobilisés dans les universités. Ces indicateurs répondent eux aussi à des injonctions paradoxales créatrices de tensions que les équipes de direction universitaire doivent gérer. C'est sur le sens des indicateurs que les présidents d'université semblent intervenir afin de se réapproprier un certain nombre d'indicateurs et les adapter au contexte de leur université.

Dans le domaine de la gestion des moyens, il en va ainsi de l'apparition des indicateurs «maîtrise des heures complémentaires » «maîtrise masse salariale sur ressources d'établissement » " temps de travail des personnels » «taux d'exécution budgétaire » qui répondent à une tension entre objectifs et moyens alloués aux universités (tableau $n^{\circ} 3$ ). L'injonction paradoxale "faire plus à moyen constant " qui provoque cette tension impose aux équipes de direction universitaire de privilégier des indicateurs de gestion de moyens (masse salariale, heures complémentaires, 
exécution budgétaire) au détriment d'objectifs de qualité du service ou de satisfaction des usagers (qualité de la gestion immobilière, disponibilité des places de bibliothèque).

De même, la substitution au sein des universités des indicateurs " coopération internationale » par l'indicateur "taux de demande de formation » révèle une concentration des préoccupations des présidents d'université sur le renforcement des projets nationaux de formation au détriment des projets internationaux. Cette substitution semble répondre à une tension entre innovation et gestion que les présidents d'université gèrent en se focalisant sur le renforcement de la gestion des formations existantes plutôt que le lancement de projets totalement innovants.

Au final, comme nous l'avons vu, notre analyse nous permet de mettre en évidence deux types de simplification qui peuvent s'expliquer par des injonctions paradoxales et tensions de gouvernance publique. La mise en évidence des tensions provoquant les simplifications des indicateurs nous permet de caractériser ces dernières dans les processus globaux de pilotage des universités (tableau $\mathrm{n}^{\circ} 3$ ). Ceci nous permet de mettre en évidence au moins trois types de processus de gestion impactés par ces tensions et ces simplifications (Formation, recherche et gestion des moyens).

En effet, au-delà du caractère instrumental de la simplification, il semble que celle-ci puisse avoir un impact sur les processus de gestion des universités et les activités qui y sont gérées. Ces simplifications instrumentales présentent de fortes dimensions processuelles tant elles peuvent, par leurs objectifs de rationalisation administrative, impacter les manières de travailler et l'exécution des services publics.

Ainsi, il apparaît que par exemple, dans les universités étudiées, la recherche est plus gérée par des simplifications quantitatives pour gérer des tensions entre ses dimensions civiques et marchandes (tensions entre rentabilité et indépendance) ou entre les spécificités de chaque université et un alignement sur les critères globaux des classements internationaux (tension entre réputation internationale et ancrage national ou régional des universités) (tableau $n^{\circ} 3$ ).

La formation est plutôt gérée à travers des simplifications quantitatives pour dépasser les tensions entre des objectifs volumiques à court terme et des objectifs stratégiques qualitatifs à long terme (tension entre objectifs et mesure, tension entre qualité et lieu d'exercice du service public).

Enfin les moyens semblent être plus gérés à travers des simplifications de sens des indicateurs afin de gérer des tensions entre les contraintes budgétaires et financières et les objectifs de qualité du service rendu (tension entre objectifs et moyens alloués aux universités, tension entre innovation et gestion).

Tableau $n^{\circ}$ 3. Simplifications des indicateurs et tensions de gouvernance

\begin{tabular}{|c|l|l|}
\hline Processus & \multicolumn{1}{|c|}{ Simplifications } & \multicolumn{1}{c|}{$\begin{array}{c}\text { Tensions de gouvernance } \\
\text { publique }\end{array}$} \\
\hline Formation & Simplifications quantitatives & $\begin{array}{l}- \text { tension entre objectifs et mesure } \\
- \text { tension entre qualité et lieu } \\
\text { d'exercice du service public }\end{array}$ \\
\hline Recherche & Simplifications quantitatives & $\begin{array}{l}- \text { tension entre rentabilité et } \\
\text { indépendance de la recherche } \\
\text { tension entre réputation } \\
\text { internationale et ancrage national } \\
\text { outégional des universités }\end{array}$ \\
\hline Moyens & Simplifications de sens & $\begin{array}{l}- \text { tension entre objectifs et moyens } \\
\text { alloués aux universités. } \\
- \text { tension entre innovation et } \\
\text { gestion }\end{array}$ \\
\hline
\end{tabular}




\section{Conclusion}

Dans un premier temps, l'identification des trois niveaux de production des indicateurs de performance universitaire (législatif, exécutif et organisationnel) nous ont permis de caractériser deux types de simplification de ces indicateurs (simplifications quantitatives et simplifications de sens). Dans un deuxième temps, le rapprochement de ces simplifications avec des tensions de gouvernance permettent de mettre en évidence les effets de ces simplifications en termes processuels.

Plusieurs apports résultent de cet article. Le premier est d'ordre heuristique. A partir du repérage de ces trois niveaux de production des indicateurs de performance universitaire, nous avons proposé une mode de caractérisation des tensions et contradictions dans les organisations qui permet de compléter les travaux de Brunsson (1993, 2002). Ceci permet d'identifier précisément les principales tensions sur lesquelles repose l'hypocrisie organisationnelle dans les organisations universitaires.

Deux enrichissements théoriques sont ensuite proposés. Un premier provient de la mise en évidence de deux niveaux de simplification (simplification quantitative et de sens) qui permet de compléter les travaux relatifs à l'instrumentation et l'appropriation des outils de gestion publique (Lascoumes et le Galès, 2012 ; Dreveton et al. op.cit. ; Boitier et Rivière, op.cit.). Un deuxième enrichissement se situe autour des simplifications que nous proposons d'illustrer à travers les trois processus les plus impactés par les tensions de gouvernance publique. Celles-ci constituent des simplifications processuelles des modalités de l'action publique qu'il importe de mettre en avant tant elles peuvent impacter, de manière significative, les simplifications administratives à l'adresse des usagers qui les précèdent ou qui leur succèdent.

Par contre, ce travail présente un certain nombre de limites empiriques et méthodologiques.

D'une part, sur le plan méthodologique, le recueil de données effectué auprès des présidents d'université malgré son exhaustivité imposera de procéder à des analyses contradictoires auprès d'autres acteurs universitaires (Doyens d'UFR, membres des conseils centraux) pour valider la robustesse de nos résultats.

D'autre part, sur le plan empirique, même si notre cadre heuristique peut ouvrir des perspectives de recommandations managériales auprès des dirigeants d'université, celui-ci nécessite sans doute des travaux complémentaires sur des études de cas spécifiques afin de tester son opérationnalité.

\section{Références bibliographiques}

Bartoli, Annie et Hermel, Philippe (2006), "Quelle compatibilité entre" orientation-client" et service public? », Politiques et management public, vol. 24, n³, p. 13-31.

Bartoli, Annie (2008), "Les outils du management public: fausses pistes et vrais outils », in Le management public en mutation, Paris : L'Harmattan, p. 73-94.

Bartoli, Annie, Mazouz, Bachir, Keramidas, Olivier et Larat, Fabrice (2012), "Éthique et performance en management public », Revue française d'administration publique, $\mathrm{n}^{\circ} 140$.

Berry, Michel (1983), «Une technologie invisible: l'impact des instruments de gestion sur l'évolution des systèmes humains », Documents $\mathrm{du}$ Centre de recherches en gestion, http ://crg.polytechnique.fr

Brunsson, Nils (1993), "The necessary hypocrisy », International Executive, vol. 35, n¹, p. 1-9.

Brunsson, Nils (2002), The organization of hypocrisy, Seconde édition, Copenhagen, Copenhagen Business School Press.

Brunsson, Nils, Geoffroy, François (2012), « Quand l'hypocrisie managériale protège l'organisation. Les apports de Nils Brunsson ", Revue internationale de psychosociologie et de gestion des comportements organisationnels, vol. 28, $n^{\circ} 46$, p. 301-315.

Boitier Marie, et Rivière Anne (2013b). «Freedom and Responsibility for French Universities: from global steering to local management ». Accounting, Auditing and Accountability Journal, vol. 26, n4, p. 616-649 
Boussard, Valérie et Loriol, Marc (2009), "Les cadres du ministère des affaires étrangères et européennes face à la LOLF », Revue française d'administration publique, ${ }^{\circ} 4$, 717-728.

Carassus, David, Favoreu, Christophe, Gardey, Damien et Marin, Pierre (2012). « La caractérisation et le management des déviances organisationnelles liées à la mise en œuvre d'une démarche de performance publique : application au contexte public local français », Management International, vol. $16, \mathrm{~N}^{\circ} 3$.

Chomienne, Hervé, Corbel, Pascal, et Denis, Jean-Philippe (2012), «Gestion de la propriété intellectuelle et organisations publiques de recherche: l'éthique à l'épreuve des objectifs de performance », Revue française d'administration publique, vol. 140, n 4, p. 677-692.

Crozier Michel (1964), Le Phénomène bureaucratique, Paris, Le Seuil

Dreveton Benjamin, Lande Evelyne et Portal Marine (2012). «Construire un outil de comptabilité de gestion au sein d'une université. Retour sur un acte manqué ». Management et Avenir, vol. 54, p. 126-144

Ezzamel, Mahmoud, Robson, Keith, et Stapleton, Pam (2012), « The logics of budgeting: Theorization and practice variation in the educational field», Accounting, Organizations and Society, vol. 37, $n^{\circ} 5$, p. 281-303.

Fabre, Pascal (2013), « Le doyen contrôleur des coûts: outils de gestion et allocation des ressources dans la gouvernance des universités», Comptabilité-Contrôle-Audit, $n^{\circ} 3$, p. 59-85.

Facal, Joseph ; Mazouz, Bachir (2014). «L'imputabilité des dirigeants publics : éléments de théorie et observations tirées de l'expérience québécoise », Revue Française de Gestion.

Fixari, Daniel et Pallez, Frederique (2007). «L'insoutenable légèreté de l'Etat-Une histoire de simplification administrative ». Politiques et Management public, vol. 25, n॰3, p. 83-100.

Huddon, Pierre André, Mazouz, Bachir (2015). «Le management public entre «tensions de gouvernance publique» et «obligation de résultats»: Vers une explication de la pluralité du management public par la diversité des systèmes de gouvernance publique », Gestion et management public, vol. $3, \mathrm{n}^{\circ} 4,7-22$.

Lascoumes, Pierre et Le Galès, Patrick (2012). Sociologie de l'action publique: domaines et approches. Armand Colin

Lorino, Philippe (2007), « Théories des organisations, sens et action: le cheminement historique, du rationalisme à la genèse instrumentale des organisations », Recherches, p. 54-71.

Mazouz, Bachir et Tardif, Marcel (2009), « À propos de la performance L'Arlésienne de la sphère publique », Management des organisations publiques, Théorie et applications, p. 31-61.

Mazouz Bachir, Garzon Cézar et Picard, Pascal (2012), «Les déviances dans les organisations publiques en quête de performance : vers une gestion prophylactique des risques de déviance », Management international, vol. 16, $\mathrm{n}^{\circ} 3$, p. 92-100.

Mériade Laurent (2013), «L'appropriation par simplification des logiques d'évaluation du NPM dans les universités francaises », @GRH, revue de l'AGRH, vol. 9, n4, p. 67-95

Mériade, Laurent et Mainetti, Nicolas (2013), «La réflexivité des acteurs face à la complexité des organisations publiques : un levier interactif du contrôle de la performance universitaire », Gestion et Management Public, vol.1, n³, p.3-23.

Mignot-Gérard, Stephanie (2008). «Gouvernance des universités ». In VanZanten, A. (dir.), Dictionnaire de l'Éducation. Paris, PUF.

Moll, Jodie et Hoque, Zahirul (2011). «Budgeting for legitimacy: The case of an Australian university ». Accounting Organizations and Society, vol. 36, n², p. 86-101.

Poel, Kevin, Marneffe, Wim, Bielen, Samantha, Van Aarle, Bas et Vereeck, Lode (2014). «Administrative simplification and economic growth: A cross country empirical study ». Journal of Business Administration Research, Vol. 3, $\mathrm{n}^{\circ} 1$, p. 45.

Reay, Trish, et Hinings, C. Robert (2009). «Managing the rivalry of competing institutional logics ». Organization Studies, vol. 30, p. 629-652. 